\title{
Study of single layer microwave absorber based on rice husk Ash/CNTs composites
}

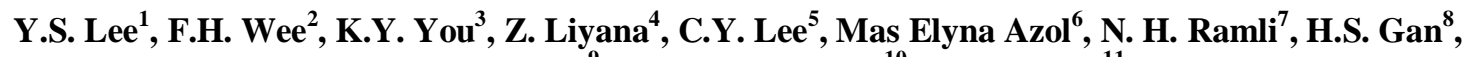 \\ M. S. Shakhirul ${ }^{9}$, Nurulbariah Idris ${ }^{10}$, E.M. Cheng ${ }^{11}$ \\ ${ }^{1,4,6,7,9.10}$ Department of Electronic Engineering Technology, Faculty of Engineering Technology, \\ Universiti Malaysia Perlis (UniMAP), Malaysia \\ ${ }^{1,2}$ School of Computer and Communication Engineering, Universiti Malaysia Perlis (UniMAP), Malaysia \\ ${ }^{11}$ School of Mechatronic Engineering, Universiti Malaysia Perlis (UniMAP), Malaysia \\ ${ }^{3,5}$ Department of Communication Engineering, Faculty of Electrical Engineering, \\ Universiti Teknologi Malaysia (UTM), Malaysia \\ ${ }^{8}$ Medical Engineering Technology Section, Bristish Malaysian Institute Universiti Kuala Lumpur, Malaysia
}

\begin{tabular}{l} 
Article Info \\
\hline Article history: \\
Received Sep 29, 2018 \\
Revised Nov 26, 2018 \\
Accepted Dec 12, 2018 \\
\hline
\end{tabular}

\section{Keywords:}

Carbon nanotubes

Microwave absorber

Relative permittivity

Rice husk ash

\begin{abstract}
This paper presents the study of microwave absorption single layer microwave absorber based on rice husk ash (RHA) with additional carbon nanotubes (CNTs) filler loading into the composites. The relative permittivity of RHA and CNTs composites (RHA/CNTs) were measured by using Agilent high temperature probe and $85070 \mathrm{E}$ software. The CST-MWS software is used to design and evaluate the microwave absorption of RHA and RHA/CNTs with metal backed plate. The microwave absorption of simulated and measured results is compared. The RHA microwave absorber only absorbed maximum, $-8 \mathrm{~dB}$ at $10.8 \mathrm{GHz}$ of the incident electromagnetic radiation and the $\mathrm{RHA} / \mathrm{CNTs}$ absorbed less than $-15 \mathrm{~dB}$ with wider bandwidth over $10.8 \mathrm{GHz}$ to $12.8 \mathrm{GHz}$ compare with RHA composites single layer microwave absorber. The results indicated that the RHA/CNTs composites have enhanced the microwave absorption of RHA composites.
\end{abstract}

Copyright $@ 2019$ Institute of Advanced Engineering and Science. All rights reserved.

\section{Corresponding Author:}

Y.S. Lee,

Department of Electronic Engineering Technology,

Faculty of Engineering Technology,

Universiti Malaysia Perlis (UniMAP), Perlis, Malaysia.

Email: yslee@unimap.edu.my

\section{INTRODUCTION}

Rapid growth in telecommunication technology is causing an increase in electromagnetic interference (EMI). EMI are uncontrolled electromagnetic waves which are harmful to human health and affect the performance of electronic devices. Therefore, microwave absorbers are required to solve the EMI related issues by reducing the EMI. Microwave absorbers are generally made of dielectric and/or magnetic materials which absorb microwave energy and convert the microwave energy to heat [1]. In recent years, many researchers are interested to use agriculture wastes that have been investigated with proven positive performance in microwave absorber applications. [2]-[4]. This is due to the unique relative permittivity of some agriculture wastes which are able to absorb microwave energy with some specific designs and composites [5], [6]. Liyana has shown that microwave absorber using sugarcane bagasse agriculture waste indicates good microwave absorption performance ( $>-10 \mathrm{~dB}$ ) with thinner absorber thickness [2]. Iqbal has reported that the single layer microwave absorber based on rice husk composite with very good microwave absorption < $-8 \mathrm{~dB}$ over 4-8 GHz [7]. In this study, carbon nanotubes material is used as additional filler composites with RHA material to enhance the relative permittivity and microwave absorption performance of RHA composite. 


\section{SINGLE LAYER ABSORBER}

Single layer microwave absorbers can be classified into impedance matching and resonant absorbers, the following discussion was about single layer absorbers that have features on both of these classifications. These features typically are an impedance matching or so called quarter wavelength resonant layers. To identify the use of those composite materials in microwave application, it is important to determine their electromagnetic properties. In dielectric absorbers, there is no magnetic loss component involved in the absorbers; hence the absorption capability of dielectric absorbers depends on relative permittivity and thickness of the single layer structure.

Testing the reflectivity characteristics of each composite meant for aiding in the design of single layered MAMs was performed using the equivalent theory shown in Figure 1 [8]. The absorption capability of a single layer is determined by dielectric losses within the material and by impedance matching conditions. On the other hand, the absorption performance is determined by the intrinsic properties of the absorbing layer, such as impedance and relative permittivity [9]. The extrinsic parameters are the thickness of absorbing layer and operating frequency. Figure 2 shows illustration of the incidence, reflecting and transmitting of the electromagnetic wave through the composite materials of single absorber.

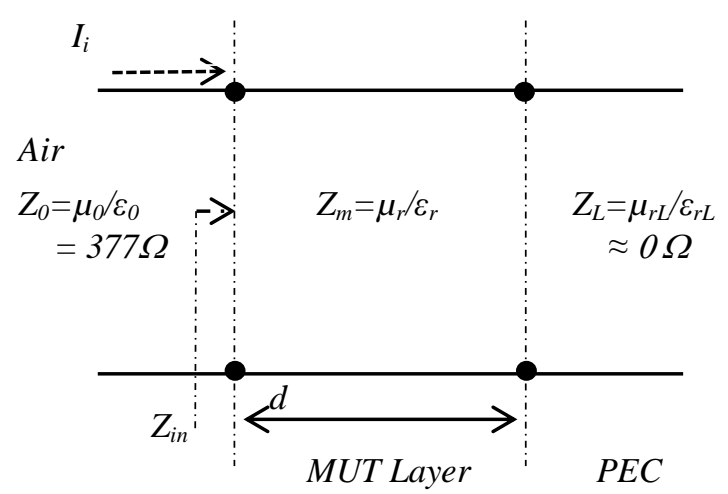

Figure 1. Microwave absorbing layer using an equivalent electric circuit explanation

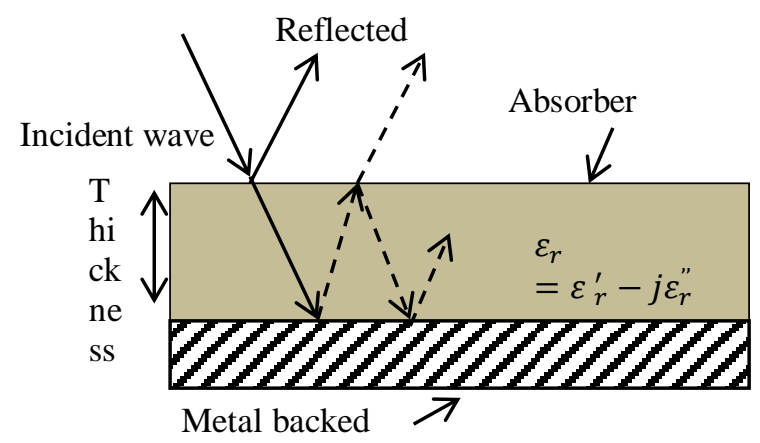

Figure 2. Illustration of the electromagnetic wave through the composite materials of single layer absorber

The air impedance assumed as free space, $Z_{0}=377 \mathrm{ohm}$, the metal back plate (PEC) as load impedance, $Z_{L}=0 \mathrm{ohm}, Z_{\text {in }}$ wave impedance at the air to absorber interface ohm, and $I_{i}$ is the current equivalent. According to the transmission line theory, the reflectivity (RL) of a metal backed single absorbing layer can be optimized with the following (1) [10]:

$$
R L(d B)=20 \log \left|\frac{Z_{\text {in }}-1}{Z_{\text {in }}+1}\right|
$$


where $Z_{i n}$, the normalized input impedance of the absorbing layer, was related to the characteristic of the material by the following (2) [11]:

$$
Z_{\text {in }}=\sqrt{\frac{\mu_{r}}{\varepsilon_{r}}} \tanh \left(j 2 \pi f d \sqrt{\mu_{r} \varepsilon_{r}}\right)
$$

where $d$ is the thickness of the absorbing layer, $f$ is the operation frequency, $\varepsilon_{r}$ is the relative permittivity, and $u_{r}$ is the magnetic properties of the material.

In order to investigate the single layer absorber consideration of the minimum RL $\left(R L_{\text {min }}\right)$ and its corresponding frequency (matching frequency, $f_{m}$ ) of various weight percentages of RHA and RHA/CNT composite is needed. $\mathrm{RL}_{\text {min }}$ decreases with increasing CNT filler loadings, which in turn leads to improved efficiency in microwave EM absorption. By decreasing the thickness of absorbers, the $\mathrm{RL}_{\text {min }}$ increases to slightly higher frequencies. This is because the microwave absorbing performance of a layer is dependent on both the microwave wavelength and the inherent characteristic of the material. If the real part of $Z_{\text {in }}$ is close to $\mathrm{Z}_{0}$ the matching condition $377 \mathrm{ohm}$ and the $\mathrm{RL}_{\mathrm{dB}}$ values $\approx-\infty$ in $\mathrm{dB}$, then, the impedance matching occurs at the frequency that equals to the matching wavelength of absorber thickness [12]. This makes the matching layer materials narrow band absorbers when the thickness of the layer satisfies (3).

$$
d_{m}=\frac{n}{4} \lambda_{m}=\frac{n}{4} \frac{\lambda_{0}}{\sqrt{\left|\mu_{r}\right|\left|\varepsilon_{r}\right|}}(n=1,3,5, \ldots .)
$$

Where $d_{m}$ is the matching thickness and $\square_{m}$ is the matching wavelength. These absorbers are made using an intermediate impedance and quarter wavelength thickness for absorption at microwave frequencies.

In this study, the optimization of single layer microwave absorbers was studied using CST-MWS software. The software settings were set to determine the reflectivity, $S_{11}$ values in $\mathrm{dB}$ for varying thickness in the frequency range 2 to18 GHz. In the parameter sweep, the template-based post processing options in CST-MWS were used to search the minimum values of the reflectivity for each value of thickness in the entire frequency sweep $2 \mathrm{GHz}$ to $18 \mathrm{GHz}$. The boundary conditions, the solver settings, the parameter sweep and design of a single layer absorber are shown in Figure 3. From the design simulation, the RL module can be evaluated by the reflectivity of the RHA and RHA/CNT single layer absorber in normal incident angle by using bi-static technique.

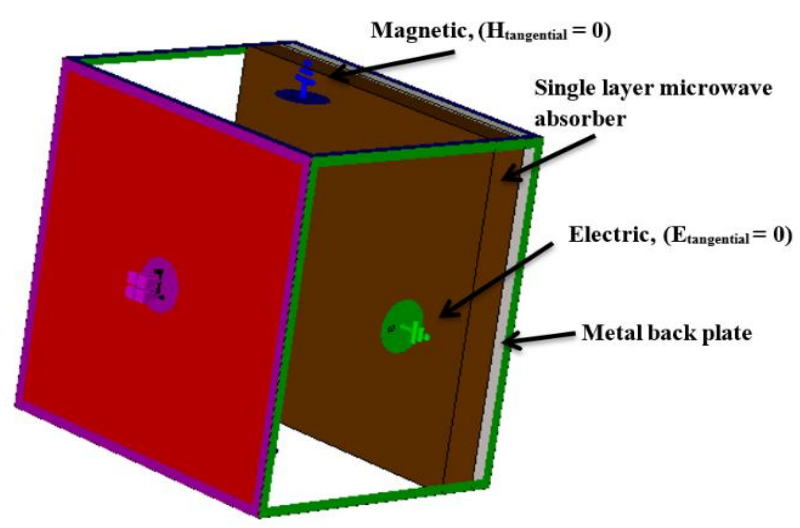

Figure 3. Single layer Absorber model and setting of the transient solver with waveguide port (red color) and boundary conditions

\section{EXPERIMENTAL}

In The single layer microwave absorbers were fabricated using different moulds with constant length, width, and thickness $(45 \mathrm{~cm} \times 45 \mathrm{~cm} \mathrm{x} 1 \mathrm{~cm})$. In this fabrication, agriculture waste $70 \mathrm{wt} \%$ weight percentage of RHA was mixed with different weight percentage of CNT (1 wt $\%, 2 \mathrm{wt} \%$, and $5 \mathrm{wt} \%$ ). Then, the polyester and $10 \mathrm{wt} \%$ of the MEKP were added to the mixture manually. After stirring the mixture, the mixture was poured into the steel mould. Figure 4 shows the fabricated single layer microwave absorber made up of the composites. 


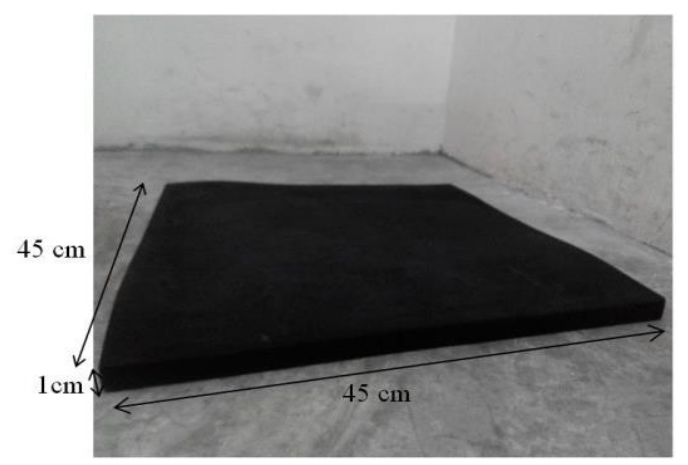

Figure 4. Fabricated single layer RHA/CNTs microwave absorber

The experiment data involve the relative permittivity over $2 \mathrm{GHz}$ to $18 \mathrm{GHz}$ was measured using an Agilent high temperature probe. The Agilent high temperature probe was connected by a coaxial cable to the Agilent performance network analyzer model E8362B with $10 \mathrm{MHz}$ to $20 \mathrm{GHz}$. The high temperature probe was contacted to the single layer microwave absorber and measured the relative permittivity of the single layer microwave absorber in ambient temperature. The relative permittivity is obtained with the average relative permittivity values from 10 points different of the single layer microwave absorber.

\section{RESULTS AND DISCUSSION}

\subsection{Effect of CNT Filler in Single Layer RHA Composites}

Based on Figure 5, the results show that the additional CNT added into the RHA composite (RHA/CNTs) have better absorption (lower reflectivity) and thinner thickness compared with RHA composites. Generally, the RHA/CNTs composite improved absorbing characteristics with a thinner matching thickness as compared with RHA composite at the same operating frequency as shown in Figure 5. The RHA composite with CNTs loading has an improved the RHA composite microwave absorption. For RHA/CNTs- 1 composites only with 1 wt $\%$ of CNTs loading in the RHA composite, the thickness of RHA/CNTs-5 absorber was $2 \mathrm{~mm}$ compared to RHA, $10 \mathrm{~mm}$ of absorber. Besides that, the RHA/CNT-5 absorber also has the lower reflectivity compared to RHA absorber at frequency range of $8 \mathrm{GHz}$ to $18 \mathrm{GHz}$, with the minimum reflectivity at $11.5 \mathrm{GHz}$ of $-27 \mathrm{~dB}$. RHA/CNTs-5 also increased in bandwidth from 10 $\mathrm{GHz}$ to $13.7 \mathrm{GHz}$ when the reflectivity was less than $-10 \mathrm{~dB}$. On the other hand, RHA/CNTs- 1 absorber with $9 \mathrm{~mm}$ thickness had minimum reflectivity, $-40 \mathrm{~dB}$ at $10.2 \mathrm{GHz}$. Such a result stresses how relative permittivity and thickness effect on the composite absorbing properties (in Section 2).

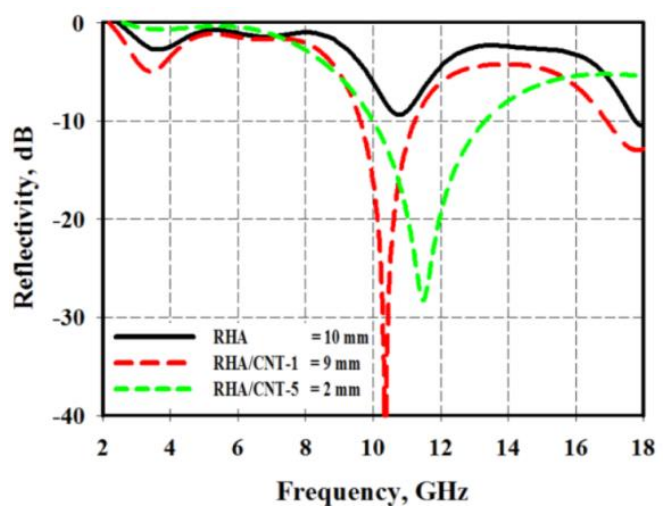

Figure 5. Comparison of the reflectivity of the RHA and RHA/CNTs single layer absorber.

In Figure 6, RHA/CNTs-5 with $1 \mathrm{~mm}, 2 \mathrm{~mm}, 3 \mathrm{~mm}, 4 \mathrm{~mm}, 5 \mathrm{~mm}$, and $10 \mathrm{~mm}$ thickness was presented. The best performance ( $\mathrm{RL}=-40 \mathrm{~dB}$ ) was observed when the thickness was $3 \mathrm{~mm}$ at $7.6 \mathrm{GHz}$. Similarly, at $4 \mathrm{~mm}$ thickness, the value of reflectivity was $-33 \mathrm{~dB}$ which was higher than the $5 \mathrm{~mm}$ thickness 
with reflectivity of $-5.2 \mathrm{~dB}$ at $5.7 \mathrm{GHz}$. However, the composite with RHA/CNTs-5 with 10 mm thickness, the reflectivity values less than $-5 \mathrm{~dB}$ for over the $2 \mathrm{GHz}$ to $18 \mathrm{GHz}$ frequency range, corresponding to $50 \%$ of the absorption of the incident wave. The RL of the RHA/CNTs-5 with $10 \mathrm{~mm}$ did not fall below $-5 \mathrm{~dB}$ because there was no matching condition between the impedance over the frequency range. This single layer absorber of RHA/CNT-5 with $10 \mathrm{~mm}$ thickness does not perform well in the tested frequency range and it also shows that an increase in thickness does not improve the microwave absorption performance. The RHA/CNT-1 and RHA/CNT-5 reflectivity results show that different thickness of single layer absorber leads to different minimum reflectivity (absorption) performance, due to different impedance matching values. Another factor that defines the obtained reflectivity curves and that might be taken into count is the relative permittivity values of the composite. For example, the RHA/CNT-1 composite has lower relative permittivity compare to RHA/CNT-5 which has a lower reflectivity less than $-10 \mathrm{~dB}$ at higher frequency over $8.4 \mathrm{GHz}$ to $17.8 \mathrm{GHz}$ with a thickness of $5 \mathrm{~mm}$ to $10 \mathrm{~mm}$. While, the RHA/CNT-5 composite with similar thickness of $5 \mathrm{~mm}$ to 10 $\mathrm{mm}$ has reflectivity less than $-10 \mathrm{~dB}$ at low frequency range $2 \mathrm{GHz}$ to $5.8 \mathrm{GHz}$.

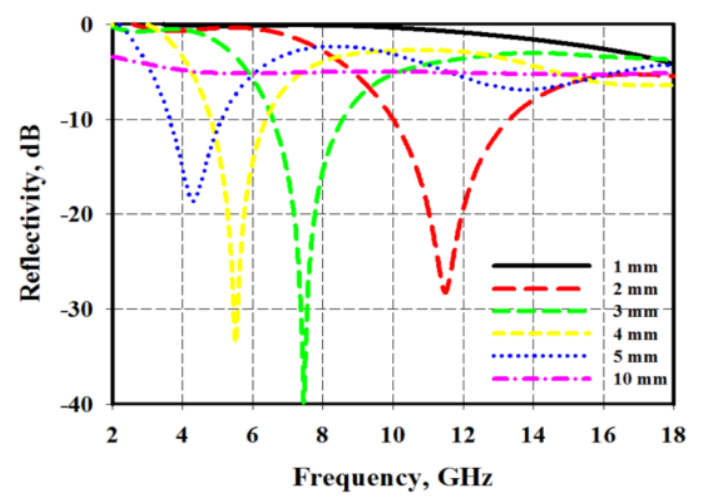

Figure 6. Effect of thickness of RHA/CNT-5 single layer absorber

\subsection{Measured and Simulated Single Layer Absorber}

In Figure 7, comparisons made between measured and simulated RHA and RHA/CNTs are presented. It can be noticed that curve indicating the simulated results shows similar behavior with respect to the curve indicating the measured results. Very good agreement is observed between the simulated and measured results. In practice, it has been noticed that the RHA/CNTs composite materials are able to absorb the electromagnetic field much better than the pure RHA composite which had analyzed. The simulated and measured results also take into account both impedance matching condition and dielectric losses of the composite material. For the RHA mixed 2 wt\% CNTs (RHA/CNTs-2), the absorption curve illustrates that the simulated reflectivity of the corresponding composites is below $-10 \mathrm{~dB}$ in the range of $2.2-3.4 \mathrm{GHz}$ and $7.8 \mathrm{GHz}$ to $9.0 \mathrm{GHz}$, whereas the measured reflectivity is slightly shifted to a lower frequency for below -10 $\mathrm{dB}$ at $2.8 \mathrm{GHz}$ to $4.0 \mathrm{GHz}$ and $8.5 \mathrm{GHz}$ to $9.5 \mathrm{GHz}$.

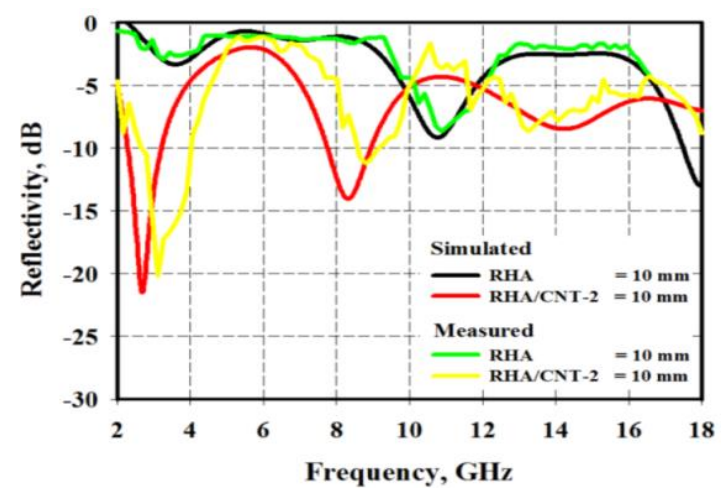

Figure 7. Comparison of reflectivity performance between measured and simulated of the RHA and RHA/CNTs single layer absorber 
In addition, the minimum measured value is $-20.4 \mathrm{~dB}$ at $3.2 \mathrm{GHz}$ while the simulated value is -21.8 $\mathrm{dB}$ at $2.6 \mathrm{GHz}$. As analyzed, the single layer absorber of RHA/CNTs composites can be used as a tuning absorber, which means shifting the operating frequency range by modifying the thickness of the absorber. When the thickness of the absorbing layer increases, the matching wavelength of the incident wave would increase. Therefore, the matching frequency will switch from a higher frequency to lower frequencies. This RHA/CNTS single layer absorber shows narrowband (resonant frequency) absorption with more than $-10 \mathrm{~dB}(\sim 90 \%)$ reflectivity in specific frequency. The incident and reflected waves resulting in a quarter wavelength reflection which allows the absorber to obtain the minimum reflected energy. For the single layer absorber, it is clearly shows that a small addition of CNTs in the composite will produce efficient fillers that increases its relative permittivity and the thickness of the single layer absorber can be reduced for specific operating frequency. The frequency of single layer absorber can be tuned within the range of $2 \mathrm{GHz}$ to 18 $\mathrm{GHz}$, by simply varying the relative permittivity and/or thickness of composite materials.

\section{CONCLUSIONS}

The microwave absorber of RHA, RHA/CNTs-1, and RHA/CNTs- 5 were optimized using CSTMWS and fabricated in single layer design. The relative permittivity of the RHA, RHA/CNTs-1, RHA/CNTs-2, and RHA/CNTs-5 were measured over 2-18 GHz. This study shows that the carbon nanotube filler added into the RHA composites increased the relative permittivity and microwave absorption performance. From the results, the RHA/CNTs-2 (9mm thickness) and RHA/CNTs-5 (2 mm thickness) of single layer absorber are thinner and better microwave absorption compare to the RHA (10 mm thickness) single layer absorber over 2-18 GHz. The additional of CNTs into the RHA composite can enhances the microwave absorption and reduce the size of single layer absorber of RHA composite. The advantage of single layer absorber is thin, light weight and able to tune the microwave absorption operating frequency.

\section{ACKNOWLEDGEMENTS}

This research was funded by the grant of Fundamental Research Grant Scheme (No. 9003-00591) under Ministry of Education, Malaysia.

\section{REFERENCES}

[1] Y. S. Lee, M. F. B. A. Malek, E. M. Cheng, W. W. Liu, K. Y. You, M. N. Iqbal, F. H. Wee, S. F. Khor, L. Zahid, and M. F. bin Haji Abd Malek, "Experimental determination of the performance of rice husk-carbon nanotube composites for absorbing microwave signals in the frequency range of 12.4-18 GHz," Prog. Electromagn. Res., vol. 140, pp. 795-812, 2013.

[2] L. Zahid, F. Malek, H. Nornikman, N. A. M. Affendi, A. Ali, N. Hussin, B. H. Ahmad, and M. Z. A. Abd Aziz, "Development Of Pyramidal Microwave Absorber Using Sugar Cane Bagasse (Scb)," Prog. Electromagn. Res., vol. 137, pp. 687-702, 2013.

[3] M. F. B. A. Malek, E. M. Cheng, O. Nadiah, H. Nornikman, M. Ahmed, M. Z. A. Abdul Aziz, A. R. Othman, P. J. Soh, A. A. A.-H. Azremi, A. Hasnain, and M. N. Taib, "Rubber Tire Dust-Rice Husk Pyramidal Microwave Absorber," Prog. Electromagn. Res., vol. 117, pp. 449-477, 2011.

[4] M. N. Iqbal, M. F. Malek, Y. S. Lee, L. Zahid, and M. S. Mezan, "A Study of the Anechoic Performance of Rice Husk-Based, Geometrically Tapered, Hollow Absorbers," Int. J. Antennas Propag., vol. 2014, pp. 1-9, 2014.

[5] L. Zahid, M. F. A. Malek, C. E. Meng, L. W. Wen, L. Y. Seng, A. Z. Abdullah, N. S. M. Noorpi, N. M. Mokhtar, and M. A. Jusoh, "Performance of Sugarcane Bagasse and Rubber Tire Dust Microwave Absorber in Ku Band Frequency," Theory Appl. Appl. Electromagn., pp. 207-214, 2015.

[6] M. N. Iqbal, M. F. B. A. Malek, Y. S. Lee, L. Zahid, M. I. Hussain, M. F. bin Haji Abd Malek, N. F. Mohamed Yusof, N. Saudin, N. A. Abu Talib, I. M Nadeem, M. Fareq, A. Malek, S. L. Yeng, Z. Liyana, M. N. Iqbal, M. I. Hussain, F. H. Mohd, A. Malek, M. Y. Nur Fairuz, and others, "A simple technique for improving the anechoic performance of a pyramidal absorber," Prog. Electromagn. Res. M, vol. 32, pp. 129-143, 2013.

[7] M. N. Iqbal, M. F. bin A. Malek, S. H. Ronald, M. S. Bin Mezan, K. M. Juni, and R. Chat, "A Study of the EMC Performance of a Graded-Impedance, Microwave, Rice-Husk Absorber," Prog. Electromagn. Res., vol. 131, pp. 19-44, 2012.

[8] K.-Y. Park, J.-H. Han, S.-B. Lee, J.-B. Kim, J.-W. Yi, and S.-K. Lee, "Fabrication and electromagnetic characteristics of microwave absorbers containing carbon nanofibers and NiFe particles," Compos. Sci. Technol., vol. 69, no. 7-8, pp. 1271-1278, Jun. 2009.

[9] Y. Zhai, Y. Zhang, and W. Ren, "Electromagnetic characteristic and microwave absorbing performance of different carbon-based hydrogenated acrylonitrile-butadiene rubber composites," Mater. Chem. Phys., vol. 133, no. 1, pp. 176-181, Mar. 2012.

[10] B. Belaabed, J. L. Wojkiewicz, S. Lamouri, N. El Kamchi, and T. Lasri, "Synthesis and characterization of hybrid conducting composites based on polyaniline/magnetite fillers with improved microwave absorption properties," $J$. Alloys Compd., vol. 527, pp. 137-144, Jun. 2012. 
[11] F. Xu, L. Ma, Q. Huo, M. Gan, and J. Tang, "Microwave absorbing properties and structural design of microwave absorbers based on polyaniline and polyaniline/magnetite nanocomposite," J. Magn. Magn. Mater., vol. 374, pp. 311-316, Jan. 2015.

[12] D. Micheli, C. Apollo, R. Pastore, and M. Marchetti, "X-Band microwave characterization of carbon-based nanocomposite material, absorption capability comparison and RAS design simulation," Compos. Sci. Technol., vol. 70, no. 2, pp. 400-409, 2010.

\section{BIOGRAPHIES OF AUTHORS}

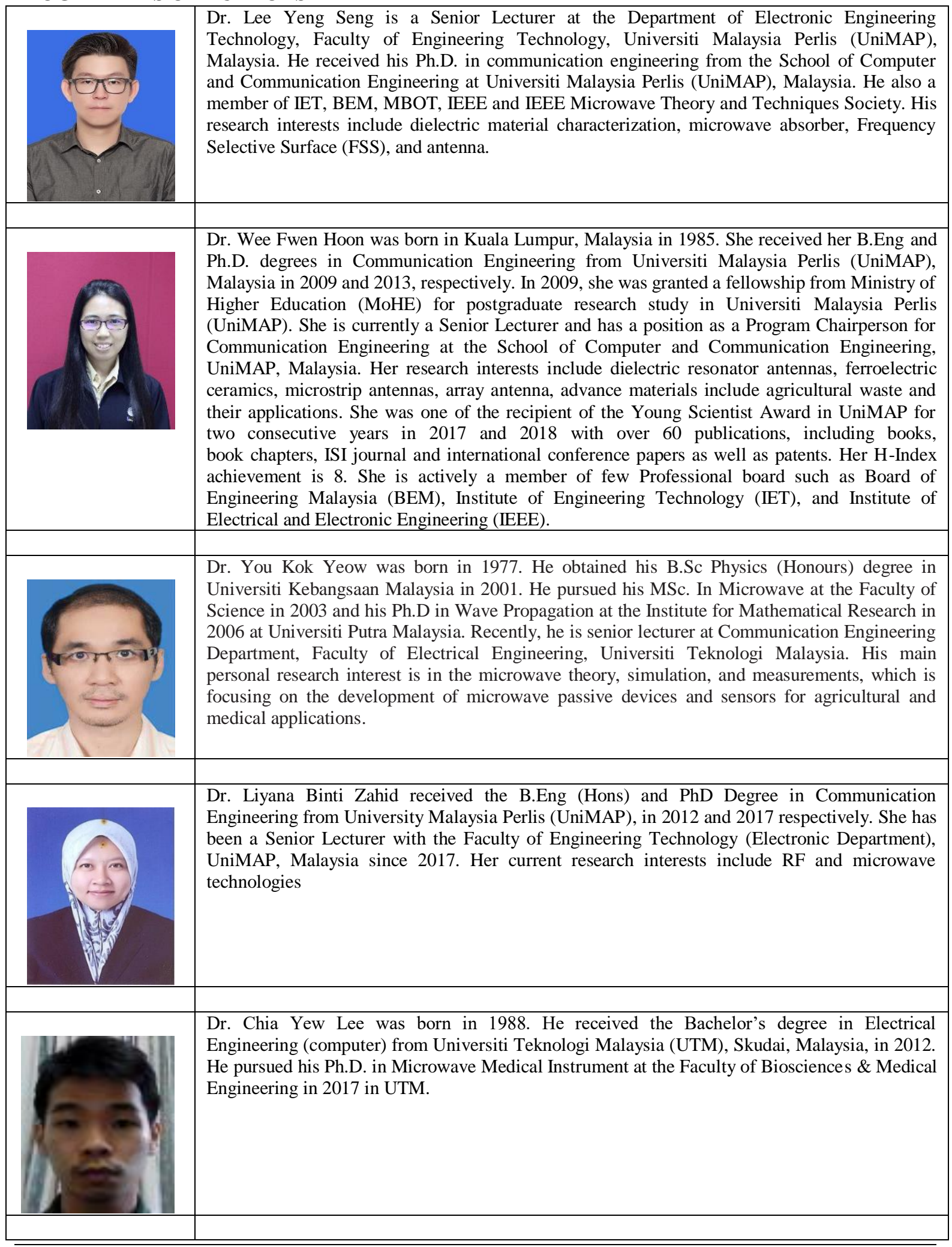




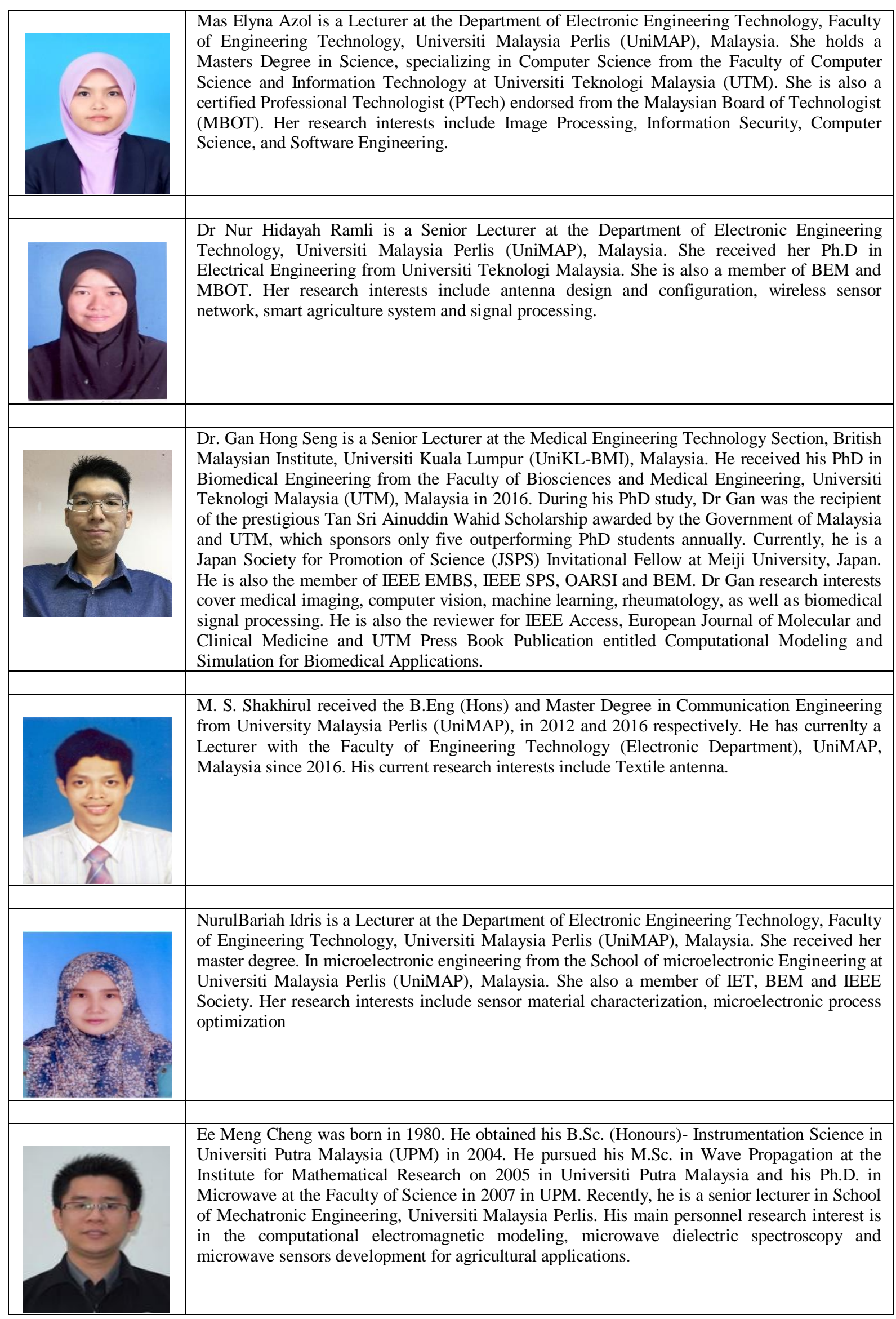

